\title{
Mechanisms of multidimensional grouping, fusion, and search in avian texture discrimination
}

\author{
ROBERT G. COOK, KIMBERLEY K. CAVOTO, and BRIAN R. CAVOTO \\ Tufts University, Medford, Massachusetts
}

\begin{abstract}
The influence of dimensional organization on pigeon texture perception was examined in a simultaneous conditional discrimination procedure. Six experienced pigeons were reinforced for pecking at a small block of target elements randomly located within a larger array of distractor elements in each texture stimulus. Target/distractor differences in color, size, orientation, and combinations of these dimensions were examined. In Experiment 1, the influence of target/distractor similarity on performance was investigated by using different forms of unidimensional and conjunctively organized texture stimuli made of two and three dimensions. Targets in feature displays, in which the two regions consistently differed along a single dimension, were located more accurately than targets in conjunctive displays, where a combination of values from all dimensions defined each region. In Experiment 2, a tradeoff between response speed and accuracy was found in the pigeons' processing of conjunctive displays. In Experiment 3, the number of distractors differentially influenced the localization of feature and conjunctive targets. Overall, the pigeons' reactions to these feature and conjunctive stimuli paralleled those of humans, suggesting that functionally equivalent mechanisms may mediate the perceptual grouping, search, and discrimination of textured multidimensional stimuli in both species.
\end{abstract}

Birds are highly visual animals. Like mammals, they form a major class of highly mobile, visually dominant, diurnal vertebrates. During their daily activities, birds behave as if they perceive an object-filled visual world similar to our own. If so, how do birds accomplish this remarkable perceptual feat, especially given the different organization and overall smaller size of the avian brain (Pearson, 1972)? We have been examining this larger comparative question by investigating the mechanisms underlying the perception and discrimination of visual textures by pigeons (Cook, 1992a, 1992b, 1992c, 1993a, 1993b). Visual textures are multidimensional, multielement patterns in which distinct global regions can be derived from the perceptual grouping of the smaller component elements (Beck, 1966, 1982; Julesz, 1981; Marr, 1982; see Figure 1 for examples). The mechanisms underlying the rapid grouping and segregation of such textured stimuli are of considerable interest, because they seem to involve many of the same processes that are essential to the perception and recognition of objects, such as boundary and edge detection, surface recognition, and figure/ ground resolution.

Among the first areas that we examined in trying to understand how pigeons process such stimuli were textured analogues of feature and conjunctive visual search displays (Cook, 1992b). These two types of stimuli are of particular interest, because they produce dramatically dif-

This research was supported by a grant from the National Science Foundation (BNS-8909040) to R.G.C. The authors thank Sal Soraci, Jeffrey Katz, and Sherrill Cook for their helpful suggestions on earlier drafts of this report. Request for reprints should be sent to R. G. Cook, Department of Psychology, Paige Hall, Tufts University, Medford, MA 02155 (e-mail: rcook@pearl.tufts.edu). ferent forms of visual search behavior in humans. In feature displays, the target is uniquely different from the surrounding distractors in a single dimension, whereas in conjunctive displays, the target is uniquely defined by a combination of dimensions partially shared with the surrounding distractors. It has been established in humans that the visual search for feature targets is rapid and independent of the number of distractors in the display, whereas the search for conjunctive targets is slower and highly dependent on the number of distractors that are present (Treisman \& Gelade, 1980). The explanation of this robust difference stands at the center of many current theories of human visual search (Cave \& Wolfe, 1990; Duncan \& Humphreys, 1989; Treisman \& Gelade, 1980; Treisman \& Sato, 1990).

In originally accounting for this search difference, Treisman and Gelade (1980) proposed that two separate processes were involved, the first being an early parallel preattentive mechanism that initially parses the visual properties of the display into separate maps of the individual features, and the second being a spatially limited attentional mechanism required to integrate these separated features into multidimensional percepts. The fast distractor-independent search for feature targets is due to the fact that they activate different feature maps from those activated by the surrounding distractor elements, allowing the parallel output of the early preattentive stage to guide responding. The slower distractor-dependent search for conjunctive targets, on the other hand, is due to the additional need to use the secondary attentional mechanism to properly fuse the multiple attributes of these targets, necessitating that the "attentional spotlight" be serially maneuvered about the display in order to correctly locate and identify them. 
Testing pigeons with feature and conjunctive texture stimuli, Cook (1992b) found that their perceptual reactions were markedly similar to our own. In a target localization task - where the birds had to locate and peck at an odd, randomly located, target region of elements within a larger array of contrasting distractor elements-the pigeons were far more accurate at finding and pecking the target regions of feature displays than those of conjunctive displays. This behavioral similarity suggested that pigeons and humans may share analogous "preattentive" processes organized into color and shape channels that independently function to perceptually group and segregate regions of visual space.

Since those initial pigeon experiments, a number of advances in the analysis of the human processing of feature and conjunctive stimuli have been made. Of most relevance for the present article are investigations of the human visual search for conjunctive targets composed of three or more relevant dimensions (Dehaene, 1989; Quinlan \& Humphreys, 1987; Treisman \& Sato, 1990; Wolfe, Cave, \& Franzel, 1989). In this research, it was argued that if a secondary attentional mechanism is required in order to conjoin the separate features of the display as Treisman and Gelade (1980) maintained, then any increase in the number of relevant dimensions to be combined should negatively affect conjunctive search. This turned out not to be the case for humans: the search for conjunctive targets created from three or more relevant dimensions was faster than that for those created from two dimensions (Dehaene, 1989; Quinlan \& Humphreys, 1987; Wolfe et al., 1989), and in some cases, it even approached that for feature displays (Wolfe et al., 1989). Consequently, the newer results suggested that the degree of feature overlap or similarity among the target and distractor elements, rather than the feature/conjunctive status of a display per se, determines the rate of human visual search. Because only two dimensions had been employed in constructing the feature and conjunctive displays used in the pigeon experiments (Cook, $1992 \mathrm{~b}$ ), the role of similarity in the pigeons' responses to the same types of displays could not be directly evaluated, provoking the experiments reported in this article.

Here we will describe three new experiments, in which we investigated how pigeons process feature and conjunctive displays composed from two and three relevant dimensions. The results extend and amplify the processing similarity suggested by our previous research, establishing three new parallels between the responses of pigeons and those of humans to feature and conjunctive stimuli. First, like that of humans, the pigeons' performance with these displays is governed by the interelement similarity of the target and distractor regions. Second, like humans, pigeons have secondary processes that are capable of deriving display information beyond what is available from the proposed preattentive grouping stage, but that operate at the cost of increased processing time. Third, like humans', the pigeons' processing of feature and conjunctive displays is affected quite differently by the number of distractors present in these displays.

\section{EXPERIMENT 1}

In Experiment 1, we investigated how pigeons performed with feature and conjunctive displays composed of two and three relevant dimensions. These displays allowed us to assess the role of interelement similarity in the pigeon's performance with these multielement displays and compare it with established human performance with conceptually similar displays (Dehaene, 1989; Quinlan \& Humphreys, 1987; Treisman \& Sato, 1990; Wolfe et al., 1989). We tested 6 pigeons that were highly experienced in a target localization task (Cook, 1992a, 1992b, 1993b). At the start of the experiment, these birds were being regularly tested with nearly 5,000 different texture displays, readily locating and pecking at any contrasting color or shape target region within a larger distractor region of textured elements (see Figure 1 for examples). The first experiment consisted of inserting, among these numerous baseline color and shape target localization trials, a series of test trials that would enable us to evaluate the birds' reactions to different forms of feature and conjunctive test displays.

In order to examine the role of similarity in the processing of feature and conjunctive displays, we varied the number of relevant dimensions involved in defining the target/distractor contrasts. Like the baseline displays, all test displays contained an odd target region of elements randomly located within a larger region of surrounding distractor elements. For the test displays, however, these two regions were made only from line elements that could potentially differ in their orientation, size, and color. The values used to form these three dimensions were carefully selected to ensure that the three dimensions' psychological contributions to the displays were as equivalent as possible. As determined by their ability to support identical levels $(80 \%)$ of target localization in preexperimental baseline tests, these values were vertical $(\mathrm{V})$ and horizontal $(\mathrm{H})$ for the orientation dimension, large (L) and small (S) for the size dimension, and green $(\mathrm{G})$ and cyan $(\mathrm{C})$ or gray (Gy) and light blue (B) for the color dimension.

The psychological status of the different test displays was manipulated by varying the dimensional attributes of the distractor elements surrounding a particular target region, whose identity was fixed across a set of test displays (see Experiment 2 of Cook, 1992b). Two equivalent sets of test displays with different targets were created for Experiment 1, one based on a target region that comprised small green horizontal (SGH) line elements, and a second based on a target region that comprised large gray vertical (LGyV) line elements. The use of these contrasting target regions prevented the pigeons from using only a particular feature or dimension to discriminate these displays. Each of these two sets of test displays contained feature and conjunctive displays composed from two relevant dimensions, feature and conjunctive displays composed from three relevant dimensions, element displays varying in only a single dimension, and redundant displays differing in two and three dimensions.

The feature and conjunctive displays composed of two dimensions varied in color and orientation and were con- 


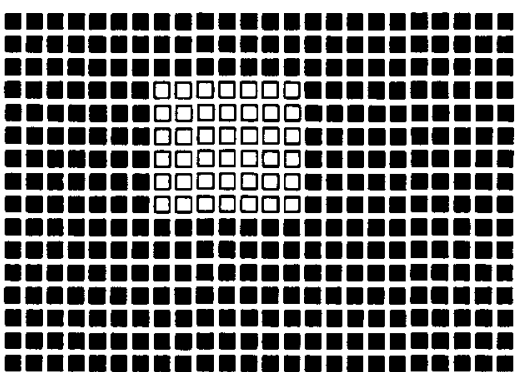

COLOR BASELINE

$\begin{array}{ll}\text { BLUE }(* 1) & \text { GRAY }(* 7) \\ \text { GREEN }(* 2) & \text { ORANGE }(* 52) \\ \text { CYAN }(* 3) & \text { ROSE }(* 37) \\ \text { RED }(* 4) & \text { LIGHT BLUE }(\text { (*25) } \\ \text { MAGENTA (*5) } & \text { YELLOW }(* 54)\end{array}$

BROWN ( $($ 20)

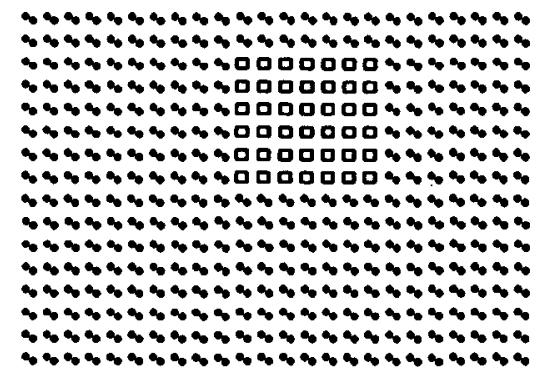

SHAPE BASELINE

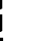

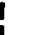 ,

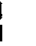 政}

.

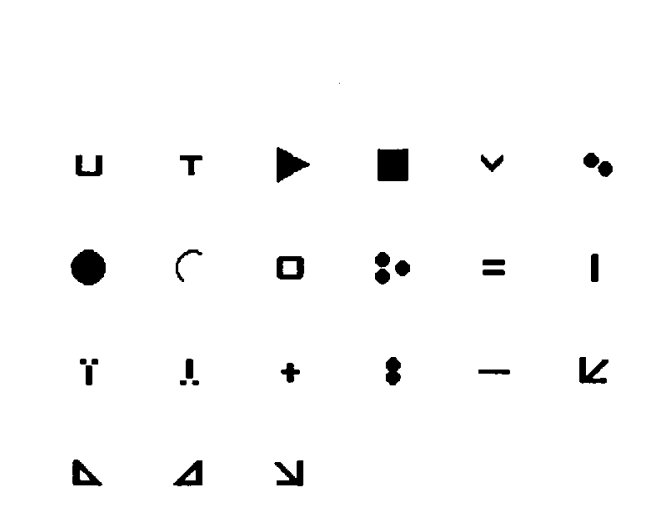

Figure 1. The left half of the figure shows examples of the baseline color and shape displays used in all three experiments. The right half lists the colors and shapes used to make the baseline displays. The VGA palette values for each color are in parentheses. The last four shapes were used only during Experiment 3.

ceptually identical to those tested earlier (Cook, 1992b). Two feature displays and one conjunctive display were made with each of the two target regions, with each target region randomly located in a distractor region formed by a random mixture of two different distractor elements. The distractor elements of the feature-color and featureorientation displays consistently differed from those of the target in either color or orientation, respectively, while those of the conjunctive display each shared a different value in common with the target. Table 1 lists the specific distractor elements used to make these displays.

The feature and conjunctive test displays composed of three dimensions were conceptually similar to those tested with humans by Wolfe et al. (1989). The additional third dimension allows three types of feature displays and two types of conjunctive displays to be formed, with the target region of each randomly located within a random mixture of three different distractor elements. For the color, size, and orientation feature displays, these distractor elements consistently differed from those of the target region in a single dimension (an example of a feature-color display appears in the upper left of Figure 2). In the 1 -shared conjunctive displays, each of the three distractor elements shared a different feature with the target region (top right of Figure 2). In the 2-shared conjunctive displays (bottom left of Figure 2), the distractor elements each shared two features in common with the target region. Table 1 lists the specific distractor elements used in making each of these displays.

Comparisons among these different types of conjunctive stimuli allowed us to investigate how similarity, as defined by the feature overlap among a display's target and distractor elements, influenced pigeon target localization. If pigeons respond on the basis of the similarity relations in the displays as humans do, then performance with the 1-shared three-dimensional conjunctive display should be better than with the 2-shared conjunctive condition or the standard two-dimensional conjunctive condition. The reason for this prediction is that each of the 1-shared conjunctive display's distractors shares only a single feature with the target, and so the average amount of overlap between its target and distractor elements $(33 \%)$ is less than that in the 2 -shared conjunctive condition $(66 \%)$ or the standard conjunctive condition $(50 \%)$. The greater dissimilarity among the elements appears to allow humans to visually search for 1-shared targets more quickly than for either 2-shared or standard conjunctive targets, despite their common conjunctive status (Dehaene, 1989; Quinlan \& Humphreys, 1987; Wolfe et al., 1989). For the same reason, similarity-based visual search theories such as guided search (Cave \& Wolfe, 1990) also predict that the standard conjunctive condition should support better performance than should the 2 -shared condition ( $50 \%$ vs. $66 \%$ ), although the evidence for this prediction has been somewhat 
Table 1

Test Display Conditions Used in Experiment 1

\begin{tabular}{|c|c|c|c|c|c|c|}
\hline \multirow[b]{2}{*}{ Displays } & \multicolumn{6}{|c|}{ Surrounding Line Elements } \\
\hline & \multicolumn{3}{|c|}{ SGH Targets } & \multicolumn{3}{|c|}{ LGyV Targets } \\
\hline \multicolumn{7}{|c|}{ Two-Dimensional Distractors } \\
\hline Feature-color & SCV & $\mathrm{SCH}$ & & LBV & LBH & \\
\hline Feature-orientation & SGV & SCV & & LBH & LGyH & \\
\hline Conjunctive & $\mathrm{SCH}$ & SGV & & LGyH & LBV & \\
\hline \multicolumn{7}{|c|}{ Three-Dimensional Distractors } \\
\hline Feature-color & SCV & LCH & $\mathrm{LCV}$ & $\mathrm{LBH}$ & SBV & SBH \\
\hline Feature-orientation & $\mathrm{SCV}$ & LCV & LGV & SBH & LBH & SGyH \\
\hline Feature-size & LCH & LCV & LGV & SBV & SBH & SGyH \\
\hline 1-shared conjunctive & LGV & SCV & LCH & LBH & SBV & SGyH \\
\hline 2-shared conjunctive & SGV & LGH & $\mathrm{SCH}$ & LBV & LGyH & SGyV \\
\hline \multicolumn{7}{|c|}{ Element Distractors } \\
\hline Color & $\mathrm{SCH}$ & & & LBV & & \\
\hline Orientation & SGV & & & LGyH & & \\
\hline Size & LGH & & & SGyV & & \\
\hline \multicolumn{7}{|c|}{ Redundant Distractors } \\
\hline \multirow[t]{3}{*}{ Two differences } & SCV & & & LBH & & \\
\hline & $\mathrm{LCH}$ & & & SBV & & \\
\hline & LGV & & & SGyH & & \\
\hline Three differences & LCV & & & SBH & & \\
\hline
\end{tabular}

mixed in humans (Quinlan \& Humphreys, 1987; Treisman \& Sato, 1990; Wolfe et al., 1989). Nevertheless, if interelement similarity is the principle factor determining the pigeons' ability to locate conjunctively defined targets, we should see the 1-shared conjunctive displays composed of three dimensions supporting the highest level of discrimination among the conjunctive displays, followed by the standard conjunctive display composed of two dimensions, and then the 2-shared conjunctive display with three dimensions. On the other hand, if solely the conjunctive relation among the elements is critical, as feature-integration theory postulates (Treisman \& Gelade, 1980), then a different pattern of results should arise. First, target localization accuracy should be poorer with all three conjunctive displays, regardless of their degree of feature overlap, than with the feature displays. Second, because of the greater number of dimensions to be integrated, performance should be poorer with the conjunctive displays composed of three dimensions than with those composed of two dimensions.

For humans, the differences among feature and conjunctive displays are typically measured in search reaction time (RT). In our target localization procedures, pigeons do not typically compensate for the difficulty of a display by taking longer to process it, but instead usually respond within $800 \mathrm{msec}$ of the onset of any texture stimulus regardless of its discriminability (Cook, 1992a, 1992b). This "speeded" discrimination behavior results in our experimental differences' usually occurring in measures of target localization accuracy rather than RT (see Pachella, 1974, for a discussion of the tradeoff between response speed and accuracy), and we expected to see in the former mea- sure the greatest effects of dimensional organization on the pigeons' performance.

\section{Method}

Subjects. Six highly experienced male White Carneaux pigeons were tested. They had been tested daily in the target localization discrimination described below for 3 years prior to this study. They werc maintained at $80 \%$ of their free-feeding weights during testing.

Apparatus. Testing was conducted in a flat-black Plexiglas chamber $(38 \mathrm{~cm}$ wide $\times 36 \mathrm{~cm}$ deep $\times 38 \mathrm{~cm}$ high). All stimuli were presented on a color computer monitor (NEC Multisync 2A; Wooddale, IL) visible through a $26 \times 18 \mathrm{~cm}$ viewing window in the middle of the front panel. The viewing window's bottom edge was $20 \mathrm{~cm}$ above the chamber floor. A thin piece of glass mounted in this window protected the monitor. Pecks to the monitor screen were detected by an infrared LED touch screen (resolution of $96 \times 48$ locations; EMS Systems, Champaign, IL) mounted behind a $40-\mathrm{mm}$-wide Plexiglas ledge that went around the inside edge of the viewing window. A $28-\mathrm{V}$ houselight was located in the ceiling and was illuminated at all times, except when an incorrect choice was made. A food hopper was located in the middle of the front panel, its access hole flush to the floor.

All experimental events were controlled and recorded with an ATclass computer. A video card (VGA Wonder; ATI Technologies, Scarborough, ON) controlled the monitor in the VGA graphics mode $(640 \times 480$ pixels). Computer-controlled relays (Metrabyte, Taunton, MA) operated the hoppers and houselight. Stimulus and event programming were done with Microsoft QuickBasic with an attached graphics library (Xgraf; Pittsburgh, PA).

\section{Procedure}

All texture displays were $18 \times 12 \mathrm{~cm}$ in size and consisted of 384 elements arranged in a $24 \times 16$ matrix at $.75-\mathrm{cm}$ intervals. Depending on their shape, these elements were $3-6 \mathrm{~mm}$ in size. The target region on each trial consisted of a $7 \times 6$ block of elements randomly located within this larger distractor matrix. 


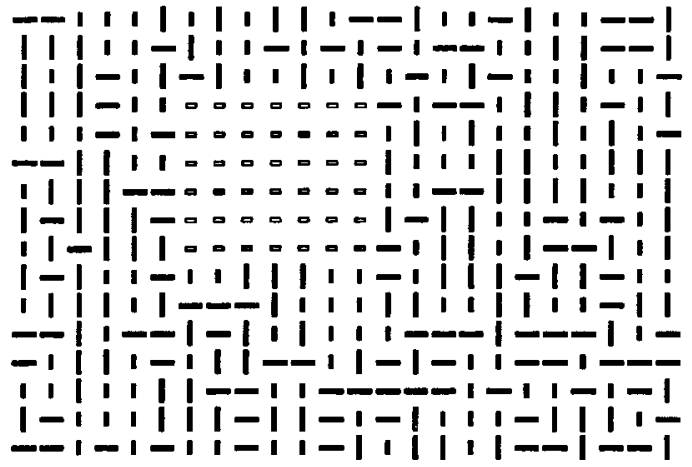

FEATURE

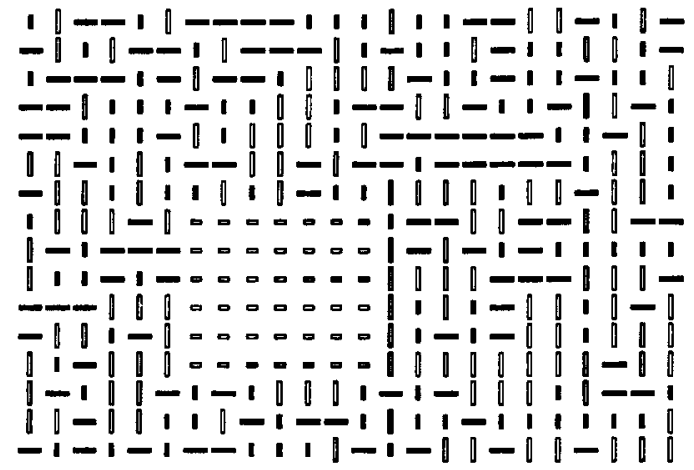

\section{1-SHARED CONJUNCTIVE}

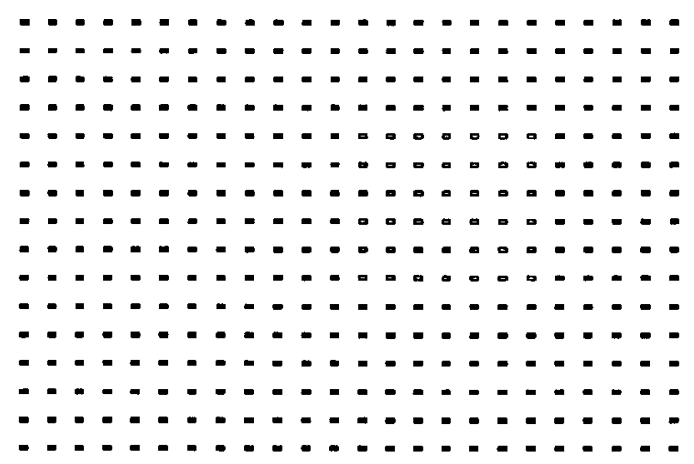

ELEMENT

\section{2-SHARED CONJUNCTIVE}

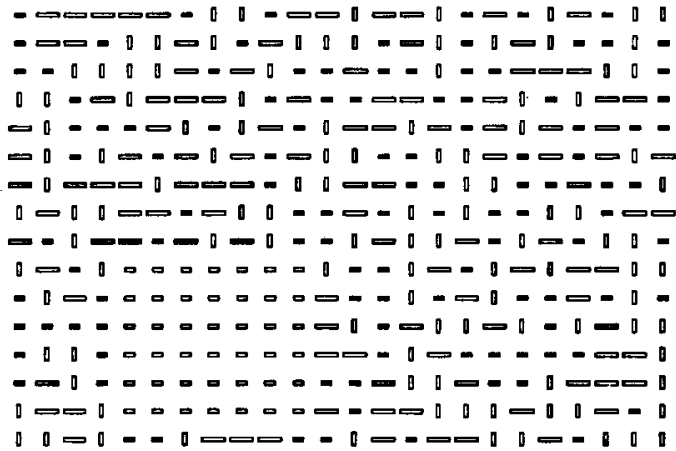

Figure 2. Examples of feature-color, 1-shared conjunctive, 2-shared conjunctive, and element-color test displays from Experiment 1.

Baseline displays. The baseline displays were created from 11 colors and 17 shapes (see Figure 1). The target and distractor elements of the 1,870 color displays differed in color but not shape (11 target colors $\times 11$ distractor colors $\times 17$ shapes minus uniform displays), whereas those of the 2,992 shape displays differed in shape but not color (17 target shapes $\times 17$ distractor shapes $\times$ 11 colors minus uniform displays). Prior to the experiment, this pool of 4,862 baseline displays was regularly being tested in 140-trial daily sessions, each composed of a random selection of 70 color and 70 shape displays from this pool.

Test displays. Test displays were made from line elements that were large $(6 \times 1 \mathrm{~mm})$ or small $(3 \times 1 \mathrm{~mm})$ in size, vertical or horizontal in orientation, and cyan (VGA \#3), green ( $\# 2)$, gray (\#7), or light blue ( $\# 25$ ) in color. These four colors were always tested in two pairs, such that (1) cyan and green and (2) gray and light blue appeared together. Test displays were created around two target regions, one of small green horizontal (SGH) line elements and the other of large gray vertical (LGyV) line elements. The feature and conjunctive test displays were then generated by surrounding these two target regions with elements varying selectively in their combination of dimensional features.

The line element distractors for the feature and conjunctive displays composed of two relevant dimensions varied in color and orientation, with their size set at the same value as the target's elements. A feature-color, feature-orientation, and conjunctive display was created with each target type. These displays were made by surrounding a target type with a randomized mixture of two distractor line elements. In feature-color displays, these distractors differed from the target in color (e.g., an S $\underline{G} H$ target region in a random mixture of SCVV and $\mathrm{SCH}$ distractors) whereas those of feature-orientation displays differed in orientation (e.g., SGH target / SGV and SCV distractors). In contrast, the distractors of a conjunctive display each shared a different feature with the target element (e.g., a $\underline{\mathrm{SGH}}$ target/ $\underline{\mathrm{SCH}}$ and SGV distractors). The positioning of these distractor elements in any display was completely randomized for every presentation.

The distractors for the feature and conjunctive displays composed of three relevant dimensions varied in their color, size, and orientation. A feature-color, feature-orientation, feature-size, 1 -shared conjunctive, and 2-shared conjunctive display was created with each target type. Each of these displays was made from a random mixture of three distractor line elements. For each of the feature displays, the distractors differed from the target in color, orientation, or size, with each sharing one or none of their remaining features with the target's elements. Each of the three distractors of the 1-shared conjunctive displays shared a different feature with that of the target; each of the three distractors of the 2 -shared conjunctive displays shared a different combination of two features with the target's elements.

In addition to the feature and conjunctive displays just described, two additional types of test displays were also tested. First, elementcolor, element-orientation, and element-size test displays were generated by surrounding each target type with a single type of distractor element that differed from the target in just its color, orientation, or size (an example of an element-color display appears in lower right of Figure 2). As will be discussed later, these element test displays were used to assess how the irrelevant dimensional variation present in the distractors of the feature displays affected the birds' discrimination of their otherwise common unidimensional contrast. Second, a set of redundant test displays was also included, whose 
target and distractor regions contrasted simultaneously in either two or all three dimensions. Four redundant displays were created by surrounding each target type with a single distractor element that differed from the target's element in color and size; color and orientation; size and orientation; or color, size, and orientation.

Thus, 30 unique test displays were generated and tested. For each target type ( $\mathrm{SGH}$ and LGyV) these consisted of two feature displays and one conjunctive display of two dimensions, three feature and two conjunctive displays of three dimensions, three element displays, and four redundant displays. Table 1 lists the specific distractors used to create these different test displays.

Discrimination testing procedures. On each baseline and test display target localization trial, the pigeon's task was to locate and peck five times at a display's target region before accumulating five pecks to any remaining portion of the display. Each trial began with a single peck to a $1.5-\mathrm{cm}$ circular white ready signal (randomly located within the upper half of the display area on each trial). This darkened the CRT screen for $2 \mathrm{sec}$, followed by the presentation of either a baseline or a test display. If the pigeon first accumulated five pecks to the area of the target, or to the immediately adjacent row and column of distractor elements around it, an accurate localization response was judged to have occurred, and the food hopper was raised for $1.8 \mathrm{sec}$. If not, the response was considered incorrect, the display was turned off, and the overhead houselight was extinguished for $10 \mathrm{sec}$. An illuminated 5-sec intertrial interval separated each trial.

Daily sessions consisted of 140 target localization trials: 30 different test displays, 108 randomly selected baseline displays ( 54 color and 54 shape), and 2 special "uniform" displays. These two uniform trials were programmed just as a standard trial, except that no visible target region was present, with each display created entirely from the repetition of randomly selected baseline element. These trials established the probability of "chance" target localization $(30.4 \%$ in Experiment 1). The order of trial presentation was randomized for each session. Twenty-four test sessions were conducted. These were grouped into four 6-session blocks for the purposes of statistical analysis. All statistical tests reported in this article were conducted with repeated measures analyses of variance (ANOVAs); $\alpha \leq .05$.

\section{Results}

In order to provide a context for judging performance with the test displays, our first analyses focused on baseline display performance. Mean target localization accuracy over the 24 sessions was significantly higher with color $(76.3 \%)$ than with shape baseline displays $[61.8 \% ; F(1,5)=$ 130.7]. Analyses of the time taken to initially peck a display (first-peck RT) revealed no significant difference between color $(727 \mathrm{msec})$ and shape $(785 \mathrm{msec})$ displays $[F(1,5)=3.8]$. Neither of these two measures of baseline performance changed significantly over the experiment $[F \mathrm{~s}(3,15)<2.9]$.

Performance with the unidimensional element test displays was examined next. Mean accuracy with the color $(83.0 \%)$, orientation $(82.7 \%)$, and size $(79.8 \%)$ element test displays was quite near the $80 \%$ value used for their preexperimental selection. These values were not reliably different from one another $[F(2,10)=1.27]$. This equality documents that the three test dimensions were equally discriminable to the birds. No significant RT differences existed among the element displays (mean RT $=713 \mathrm{msec}$ ). Target type (SGH vs. LGyV test displays) also did not significantly influence performance. Target type, in fact, had no significant effect in any analyses conducted for Experiment 1 . It will not be discussed further.
Performance with the color, size, and orientation feature displays of the three-dimensional set was highly similar to that observed with the simpler element displays. Average accuracy for the color $(80.5 \%)$, orientation $(82.5 \%)$, and size $(77.7 \%$ ) feature displays was also near $80 \%$, and these were not statistically different from one another $[F(2,10)<1]$. The same was true of the color $(80.1 \%)$ and orientation $(80.5 \%)$ feature displays composed from two dimensions $[F(1,5)<1]$. No significant RT differences were found among these feature displays (mean RT $=692 \mathrm{msec}$ ). Since there were no accuracy differences among the feature displays, subsequent analyses used separate composite accuracy scores for the two-dimensional (color and orientation) and threedimensional (color, size, and orientation) sets to summarize feature display performance in each case.

As for the comparison of feature and conjunctive test display performance, Figure 3 displays mean accuracy for the two- and three-dimensional variations of these displays. Although searching for the same targets in each case, the pigeons consistently found the feature displays easier to discriminate than the conjunctive displays. The one exception to this general conclusion was the 1-shared conjunctive display, where localization accuracy approached that observed for the feature displays.

Considering performance with the three-dimensional set first, mean accuracy was $80.2 \%$ with the combined feature displays, $76.9 \%$ with the 1 -shared conjunctive displays, and $57.6 \%$ with the 2 -shared conjunctive displays. An ANOVA (display type $\times$ block) confirmed that these differences were reliable $[F(2,10)=23.1]$. This ANOVA also revealed that accuracy with the test displays had significantly increased during the experiment $[F(3,15)=$ 26.4 , but that this increase did not interact with the display type $[F(6,30)<1]$. Pairwise comparisons further revealed that there was no significant difference between the feature and 1 -shared conditions $[F(1,5)<1]$ and that both of these conditions supported significantly better localization accuracy than did the 2-shared condition [feature vs. 2 -shared, $F(1,5)=33.7 ; 1$-shared vs. 2 -shared, $F(1,5)=$ 23.1]. RT analyses yielded no significant differences between the feature $(645 \mathrm{msec})$ and 1-shared displays [696 msec; $F(1,5)=1.2]$. Responding to the more difficult 2 -shared displays was somewhat slower $(796 \mathrm{msec})$, but this was significant only in comparison with the feature condition $[F(1,5)=6.7]$. No significant changes in RT occurred over the experiment with this set of displays $[F(3,15)<2.7]$.

Performance with the two-dimensional feature and conjunctive displays followed the same pattern, with an important exception. Accuracy was again significantly better with the feature displays $(80.3 \%)$ than with the conjunctive displays $[65.2 \% ; F(1,5)=21.1]$. Furthermore, test display accuracy generally improved over the experiment $[F(3,15)=$ 25.3], but unlike with the three-dimensional set, this improvement significantly interacted with the difference between feature and conjunctive accuracy $[F(3,15)=8.5]$. This interaction was due to a decrease in the accuracy difference between display types across the four six-session 


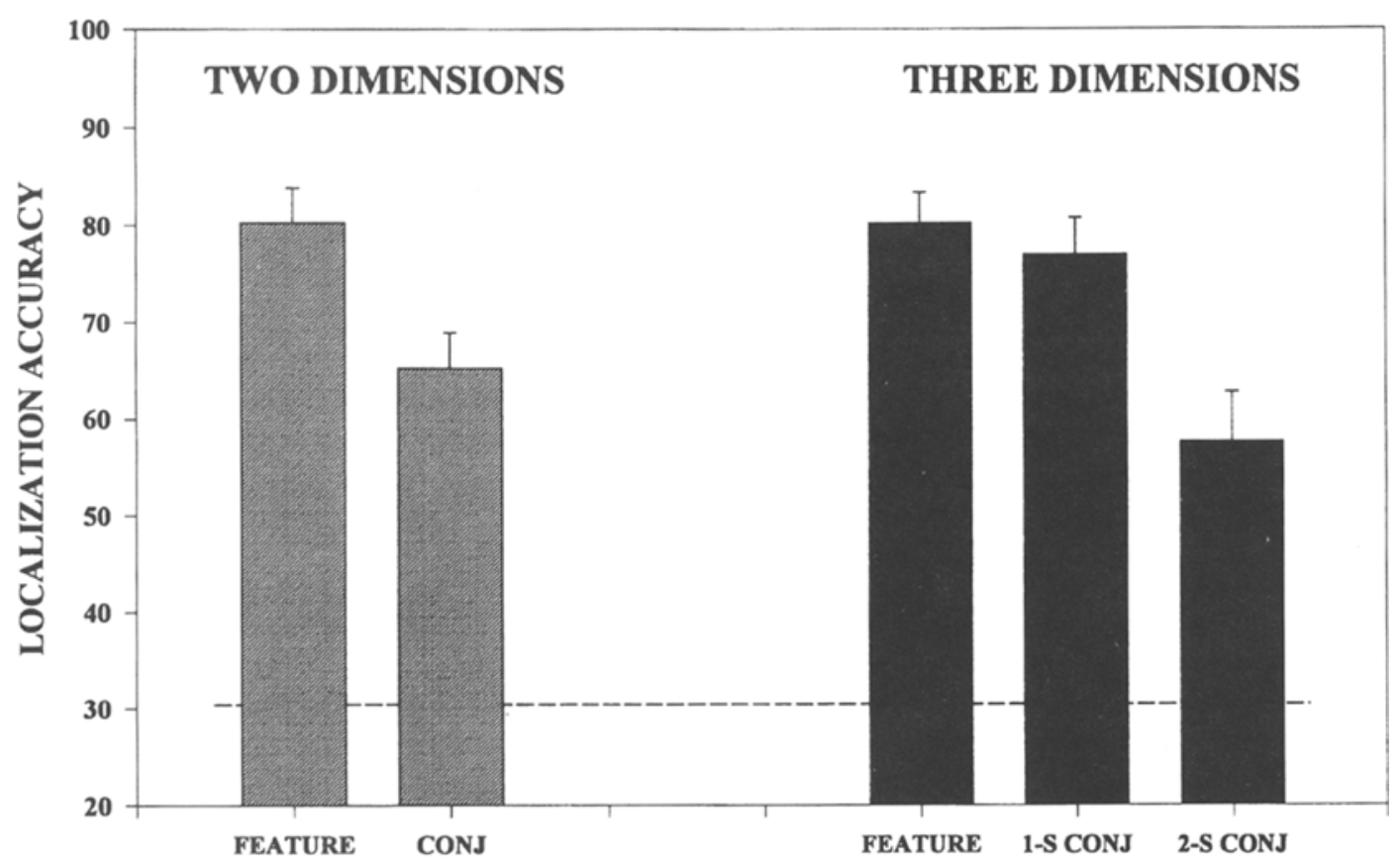

TYPE OF TEST DISPLAY

\begin{abstract}
Figure 3. Mean target localization accuracies in Experiment 1 for the 6 pigeons tested with the two- and threedimensional sets of feature and conjunctive displays (CONJ = conjunctive display; 1 -S CONJ $=1$-shared conjunctive; 2-S CONJ = 2-shared conjunctive). The dotted line represents chance performance as determined by uniform probe trials during the experiment.
\end{abstract}

blocks. During the first block, for instance, the difference between the feature $(63.2 \%)$ and conjunctive $(30.5 \%)$ conditions was both large and highly reliable $[F(1,5)=$ 54.8]; but by the last block, this difference had diminished considerably (feature $=91.5 \%$, conjunctive $=84.6 \%)$ and was no longer reliable $[F(1,5)=3.6]$. Interestingly, this change in accuracy was also accompanied by a change in the pigeons' RTs to these displays. Again during the first block, when the feature/conjunctive difference had been its largest, mean RTs to the feature (694 msec) and conjunctive $(684 \mathrm{msec})$ displays were equal and statistically unreliable $[F(1,5)=2.5]$. By the last block, when the feature/ conjunctive difference had become much smaller, mean RTs to the conjunctive displays had increased $(803 \mathrm{msec})$ and were now reliably longer than those to the feature displays [ $685 \mathrm{msec} ; F(1,5)=64.8]$. These correlated shifts in accuracy and RT suggest that by the end of the experiment the birds may have begun to trade slower response speed for increased accuracy with the two-dimensional conjunctive displays. In light of this finding, we reexamined the three-dimension and baseline display results for evidence of similar response shifts. While the trends in these data were in the same direction (slower RTs and increased accuracy in the latter stages of the experiment), they were small and had no statistical foundation.

We next compared performance among the three conjunctive displays. As already mentioned, the 1 -shared conjunctive displays $(76.9 \%)$ supported significantly more accurate target localization than did the 2-shared displays
$(57.6 \%)$. Comparison of the two-dimensional conjunctive $(65.2 \%)$ and the 1 -shared display confirmed that the latter display supported a significantly higher level of accuracy also $[F(1,5)=11.9]$. This comparison is somewhat complicated by the speed/accuracy tradeoff observed with the two-dimensional displays. Accounting for this tradeoff by looking for accuracy differences early in the experiment and RT differences in the latter stages did nothing to change this assessment. Performance with the 1-shared displays was superior either way, supporting significantly higher accuracy in the first half of the experiment $(65.8 \%$ vs. $47.3 \%)$ during which their RTs were equal, and significantly faster responding in the second half ( $717 \mathrm{vs.} 860 \mathrm{msec}$ ) when their accuracies were closer in value. Comparisons of the 2-shared conjunctive and two-dimensional conjunctive displays revealed no significant differences in accuracy $(57.6 \%$ vs. $65.2 \%$ ) or RT (796 vs. $831 \mathrm{msec}$ ) among these displays.

Two final analyses were conducted to look at the effects of irrelevant dimensional variation and dimensional redundancy on performance. The first of these involved comparing color, size, and orientation element display performance with color, size, and orientation feature display performance. This was done to see whether the irrelevant dimensional variation present in the feature displays, but not in the element displays, interfered with the discrimination of each display's relevant unidimensional contrast. Collapsed across the three-dimensions, element $(81.8 \%)$ and feature $(80.2 \%)$ test display accuracy was equivalent $[F(1,5)<1$; display (element/feature) $\times$ test block ANOVA]. We last compared 
the birds' performance on the test displays having 1 (element), 2, or 3 redundant relevant target/distractor contrasts. This analysis revealed that as the number of dimensional differences increased, so did the speed and accuracy of target localization. Collapsed over the entire experiment, performance for displays with 1,2 , or 3 relevant contrasts exhibited significant increases in accuracy [mean values of $81.8 \%, 86.4 \%$, and $88.9 \% ; F(2,10)=5.3$ ] and significant decreases in RT [727, 651, and $658 \mathrm{msec} ; F(2,10)=6.5]$.

\section{Discussion}

Experiment 1 established four important results about the abilities of pigeons to perceive and process information from multidimensional textured displays. First, feature organizations supported better target localization than did conjunctive organizations. Second, the similarity between the target and distractor elements determined accuracy within the different conjunctive organizations, with both the number of shared features (1-shared three-dimensional $>2$-shared three-dimensional) and distinctive features (1-shared three-dimensional > 1-shared two-dimensional) affecting performance. Third, evidence of a speed/accuracy tradeoff was detected in the processing of at least one type of conjunctive display. Fourth, irrelevant dimensional variation did not interfere with the birds' ability to discriminate unidimensional regional contrasts (feature $=$ element).

The results with the feature and conjunctive displays both replicate and extend those reported earlier. They clearly establish that pigeons, like humans, find feature organizations far easier to discriminate. Moreover, the pigeons' ranking of the difficulty of the feature, 1-shared, and 2-shared conjunctive displays composed of three dimensions as ordered by accuracy is identical to that of humans in visual search experiments as ordered by RT (Dehaene, 1989; Quinlan \& Humphreys, 1987; Treisman \& Sato, 1990; Wolfe et al., 1989). Even the relative size of these differences closely resembles that of previous findings for humans, with the pigeons' almost feature-like performance in the 1-shared conjunctive condition (Wolfe et al., 1989), followed by a large and considerable drop in performance in the 2-shared condition (Dehaene, 1989; Quinlan \& Humphreys, 1987; Treisman \& Sato, 1990; Wolfe et al., 1989). This strong correspondence adds further weight to the argument that the perception and processing of textured multidimensional information is highly similar in pigeons and humans.

As applied to pigeons, these results are inconsistent with feature integration theory (Treisman \& Gelade, 1980). Recall that this theory predicts that the discrimination of the 1 -shared conjunctive display should have been poorer than the discrimination of the standard two-dimensional conjunctive display, and that feature display performance should have been much better than performance with any form of conjunctive display. Neither of these predictions was supported, since accuracy with the 1-shared conjunctive displays was both superior to accuracy in the other conjunctive conditions and virtually equal to feature performance. The results instead are more consistent with the similarity-based accounts of the feature/conjunctive difference suggested by guided search (Cave \& Wolfe, 1990) or search similarity theory (Duncan \& Humphreys, 1989), in which target/distractor similarity is the more important factor in determining a display's discriminability.

While the organization of our feature and conjunctive displays was designed to directly mimic that in human visual search experiments, it was constrained by the nature of textured stimulus discrimination already performed by our highly trained avian psychophysical observers. One consequence of this restriction was that the regional contrasts of the test displays had differences besides those associated with their dimensional organization, the most obvious of which was the more homogeneous appearance of the target region relative to the distractor region. This ancillary target/distractor difference may have indeed contributed to the birds' target localization (Cook, 1993b; Honig, 1991), but it cannot by itself account for the differences among the test stimuli, because this factor was present to the same extent in all of them. In addition, our earlier research found little or no difference in the size of the feature/conjunctive effect produced by displays organized like the present ones in comparison with analogous displays that were equally heterogeneous in their element arrangement (Cook, 1992b). Nonetheless, experiments with pigeons trained to visually search for a single-element target, rather than the homogeneous region required here, would be desirable. We are currently training pigeons to perform this task, and we are optimistic that the pattern of results will be consistent with those observed with these textured variations of feature and conjunctive displays.

Turning for just a moment to a closely related, but separate, issue, it should be pointed out that these birds responded to the size, color, and orientation dimensions of the test displays as if they were perceptually separable dimensions (Garner, 1974, 1976; see Riley \& Leith, 1976, for a discussion of Garner's ideas applied to animals). This conclusion is drawn from the failure of the irrelevant dimensional variation present in the feature displays to interfere with the birds' discrimination of its relevant unidimensional contrast, as compared with their performance with the otherwise equivalent element displays. This comparison of feature and element display performance is essentially analogous to the conditions used to evaluate dimensional separability in a dimensional filtering task (Ashby, 1992; Garner, 1974; Maddox, 1992; Melara \& Marks, 1990). In such filtering tasks, separable dimensions show no ill effects of irrelevant dimensional variation in compound stimulus discriminations that require the subject to ignore this variability, whereas compounds created from integral dimensions exhibit very strong interference effects in the same setting. This difference has suggested that separable dimensions allow selective attention to the component dimensions of compounds, whereas integral dimensions maintain a "unitary" linkage that cannot be easily filtered and ignored.

It is the comparable absence of irrelevant interference in the processing of the feature displays that suggests that color, orientation, and size be classified as independent perceptually separable dimensions for birds (see Pashler, 1988, and Treisman, 1988, for the application of similar reason- 
ing to human visual search behavior). Given the unpredictability of the relevant dimension's identity from trial to trial during a session, the rapidity of the pigeons' responses, and the absence of irrelevant interference, the present outcomes also suggest that the discrimination of these dimensional contrasts is the product of a parallel visual process, rather than any attentional-like successive operation that scans across the different dimensions for a target. Consequently, in something highly akin to the human phenomenon of visual "pop-out," pigeons also seem able to immediately sense the presence of unidimensional contrasts in these displays regardless of their featural origin.

\section{EXPERIMENT 2}

The possible contribution of top-down search processes to the pigeons' discrimination of these textured displays was also suggested by the results of Experiment 1. Evidence of this possibility came from the correlated changes in accuracy and RT with two-dimensional feature and conjunctive stimuli over the experiment. These data, although confined to only a subset of the displays, suggest that pigeons too benefit from more slowly or extensively processing difficult stimulus displays by using additional search or identification mechanisms that help to compensate for the perceptual difficulty of discriminating conjunctively organized textures.

We suspected that a key difference between Experiment 1 and our earlier experiments in which no comparable RT differences were detected (Cook, 1992b) might be found in the number of targets appearing in the feature and conjunctive conditions. Only two different targets were used to form the feature and conjunctive displays of Experiment 1 , whereas combinations of 64 different elements were employed to create the targets of the earlier experiments. Perhaps the smaller number of targets in Experiment 1 allowed the birds to learn what to "look for" in the test displays, thus increasing their search persistence.

In Experiment 2, we investigated how the number and predictability of the targets in the test displays influenced performance. For an extended number of sessions, the same target (e.g., SGH) was repeatedly used to generate all of the test displays. Performance in these single-target sessions was then compared with that observed during multiple-target sessions, in which eight different targets (e.g., SGH, LCV, SCH, etc.) were unpredictably employed across sessions to generate the test displays. We hypothesized that repeated experience with the same target in the test displays might allow the birds to learn what to search for, possibly resulting in longer search times with the difficult conjunctive displays. Alternatively, testing with a larger unpredictable set of targets would hinder the birds' ability to use this target-specific information, resulting in the rapid target localization responses more often observed in these texture discrimination procedures. To examine these possibilities, three blocks of single-target sessions in which all test displays contained the same target were alternated with three blocks of multiple-target ses- sions in which eight different targets were used across sessions to make the test displays.

\section{Method}

Subjects and Apparatus. The same pigeons and apparatus were used.

Procedure. The same procedure, dimensions, and values were used to make the baseline and test displays as in Experiment 1, except that the gray/light-blue color pair was not used to form any of the test displays. Again, feature and conjunctive displays composed from two and three dimensions, element, and redundant displays were tested (15 total displays). In addition, three further test displays were introduced. The regions of these three feature-like displays differed in size, orientation, or color, respectively, but used a random mixture of all four possible distractors in surrounding the fixed target type. Performance was $3 \%-4 \%$ less accurate with these displays than with the other feature displays. Except to note their potential contribution to the learning of the repeated target's identity during single-target sessions, however, these displays will not be discussed further, since they were added for reasons not germane to this article.

Each daily session consisted of 140 target localization trials, consisting of 120 randomly selected baseline trials (60 color and 60 shape displays), 18 test display trials, and 2 uniform trials as in Experiment 1 . The order of trial presentation was randomized for each session. The organization, temporal parameters, and contingencies of reinforcement for each of these trials were also the same as in Experiment 1. As derived from performance with the uniform displays, the probability of a "chance" target localization was $28.4 \%$ in Experiment 2.

The experiment consisted of three alternations between singletarget and multiple-target testing conditions. Each single-target condition involved using a single target type to make all test displays for 16 consecutive sessions. Each of the three 16-session single-target blocks in the experiment tested a different target (SGH, LCV, and LGH elements, respectively). The multiple-target conditions conducted in between these single-target blocks involved using a different target each session to form the test displays. All eight possible target regions (SGH, SGV, LGH, LGV, SCH, SCV, LCH, LCV) were tested in a random order in 8-session blocks. The first two multiple-target segments lasted 8 sessions each; the third lasted a total of 32 sessions, consisting of four 8-session blocks.

\section{Results}

Figure 4 shows the birds' combined accuracy with feature and conjunctive test displays, grouped into 4-session blocks, across the three alternations of single-target and multiple-target testing. The different target manipulations in the test displays did not affect baseline display accuracy (the dotted line in Figure 4), which remained constant throughout the experiment $($ mean $=69.9 \%)$. Test display accuracy, in contrast, changed rather dramatically as a function of these manipulations. Test display accuracy (feature and conjunctive displays combined) significantly increased within the single-target blocks $[F(3,15)=61.5]$. This increase was smallest in the first block, most likely because of the birds' residual familiarity with the SGH target from Experiment 1. As discussed in more detail later, this increasing accuracy was also associated with significant changes in the birds' RTs during single-target blocks $[F(3,15)=5.7]$. For the multiple-target blocks, combined accuracy with the feature and conjunctive test displays was generally lower and changed little within each of these blocks. This was best evidenced in the last block, where 


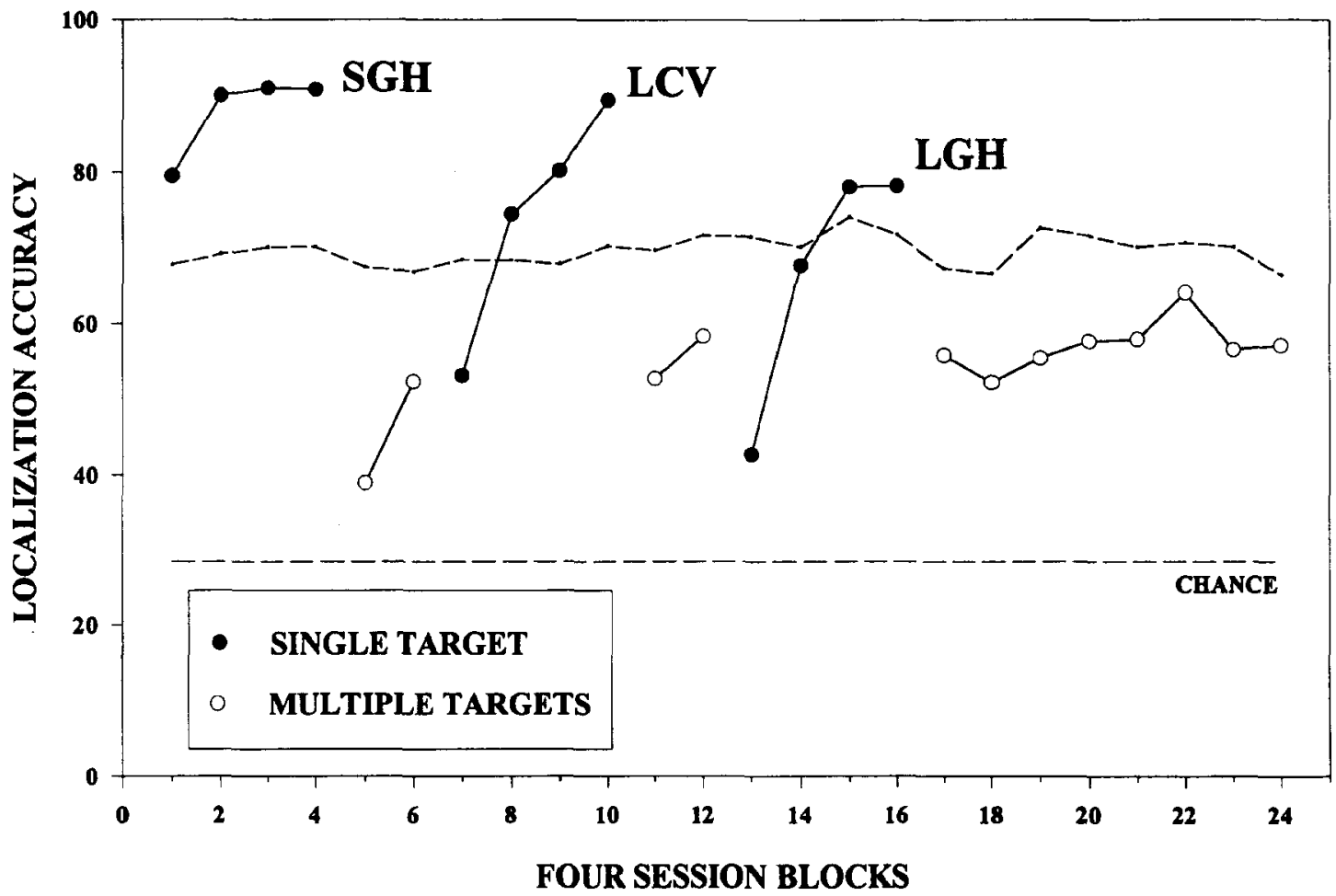

Figure 4. Mean target localization accuracies for the three single-target (filled circles) and three multiple-target (unfilled circles) phases of Experiment 2. The labels designate the target type tested in the test displays during each singletarget block. The upper dotted line represents baseline display performance over the experiment. The bottom line represents chance performance as determined by the uniform probe trials.

neither test display accuracy nor RT significantly changed over 32 continuous sessions of testing [both $F_{\mathrm{s}}(7,35)<1$ ], ruling out the possibility that the increased accuracy within the single-target blocks was due to their greater length in comparison with the first or second multiple-target block.

We next compared asymptotic performance in the singleand multiple-target conditions in order to examine in more detail the cumulative effects of these manipulations on feature and conjunctive display processing. For the multipletarget condition, we used data from just the last 32-session block, because it represented the longest sustained period of testing for this condition. For the single-target condition, we used the data from only the last 4 sessions of each single-target block, since they best represented the accumulated effects of target repetition.

Feature and conjunctive multiple-target performance. During the multiple-target test phase, performance with the feature and conjunctive displays was highly similar to that in Experiment 1, with feature organizations supporting better accuracy than did conjunctive ones, with little or no difference in RTs among these conditions. These outcomes are displayed in the top two panels of Figure 5, where the left panel displays mean accuracy and the right panel displays mean RT for these conditions. For the twodimensional set, localization accuracy was significantly better with the feature (color and orientation combined) than with the conjunctive organizations $[F(1,5)=39.5]$, and there was no significant difference in their RTs $[F(1,5)<1]$. For the three-dimensional set, feature display accuracy (color, size, and orientation combined) was also significantly better than with the conjunctive displays $[F(2,10)=$ 17.6], with again no significant differences in RT among these conditions $[F(2,10)=2.1)]$.

Feature and conjunctive single-target performance. The cumulative effects of the single-target manipulation on these same display conditions and measures are displayed in the lower two panels of Figure 5. Compared with multiple-target performance in the upper panels, accuracies were higher and response times longer at the end of the single-target blocks, with the greatest changes associated with the conjunctive displays.

For the two-dimensional set, there was now no accuracy difference between feature and conjunctive displays $[F(1,5)$ $<1$ ]; instead, a significant RT difference had emerged in its place $[F(1,5)=10.77]$. A similar pattern of responding was exhibited with the three-dimensional set as well, although the tradeoff between response speed and accuracy was not as complete as with the two-dimensional displays and was primarily associated with only the difficult 2-shared conjunctive displays. An examination of performance with the 2-shared conjunctive displays over the four panels reveals that both accuracy and RT did considerably increase during single-target testing. However, this increased accuracy never reached the same level as that with the fea- 

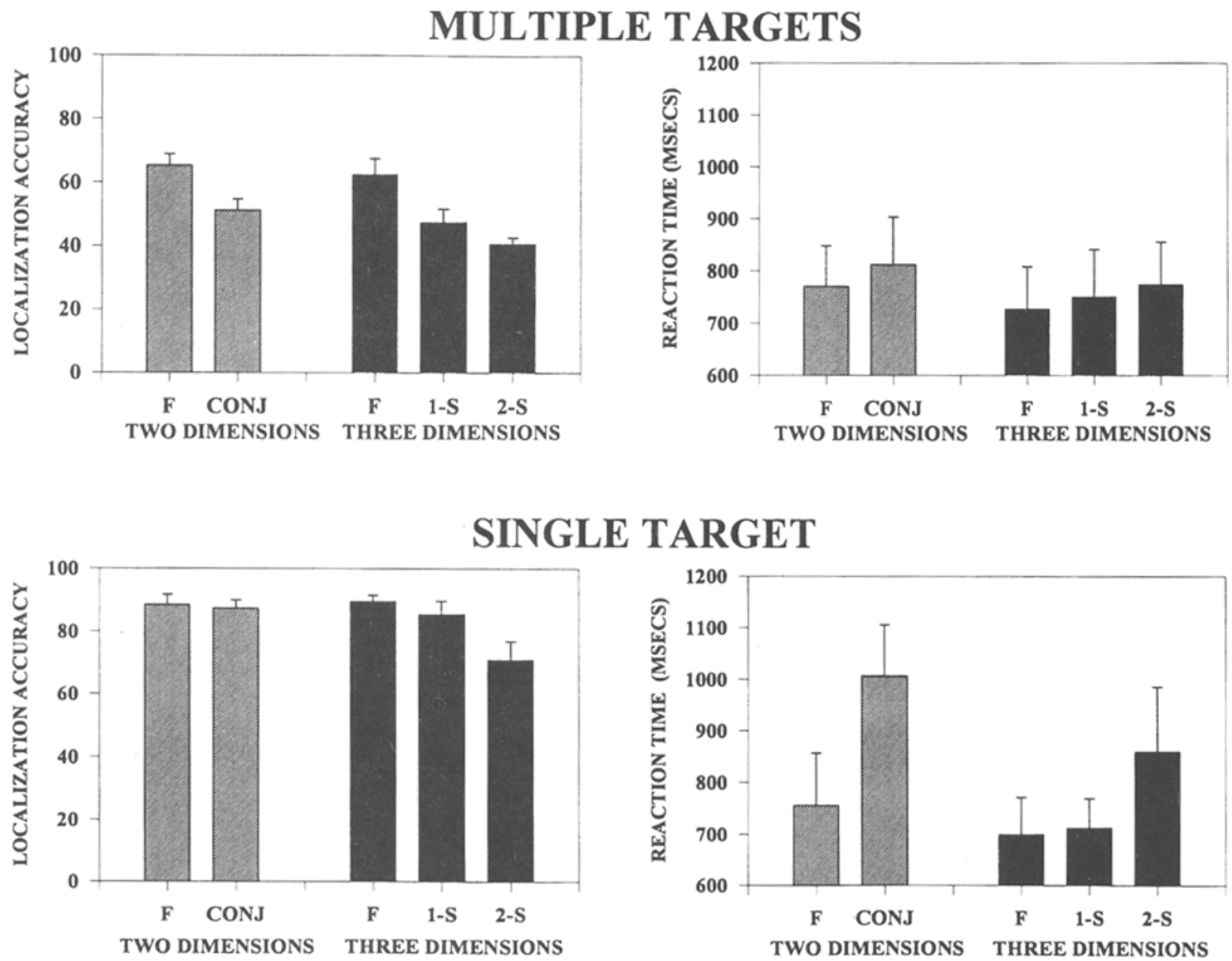

Figure 5. Mean target localization accuracies and reaction times for feature and conjunctive test displays in Experiment $2(F=$ feature; $C O N J=$ standard conjunctive; $1-S=1$-shared conjunctive; 2 -S $=2$-shared conjunctive). The top two panels show performance with the test displays during the last multiple-target phase. The bottom two panels show performance from the last four sessions of each of the three single-target blocks.

ture displays, as was the case with the two-dimensional stimuli, but, perhaps revealingly, the increase in RT was not as dramatic either. Pairwise comparisons among the feature, 1-shared, and 2-shared displays showed that the two former conditions supported both significantly higher accuracy and faster RTs than did the 2-shared displays [all $\left.F_{\mathrm{s}}(1,5)>8.2\right]$. No differences in accuracy or RT were found between the feature and 1-shared conjunctive displays $[F \mathrm{~s}(1,5)<1]$. Collectively, these results suggest that the pigeons' responses to the 2-shared displays fell somewhere in between the fast accurate processing of the feature displays and the slower, but more accurate, processing of the two-dimensional conjunctive displays. Thus, while the pigeons were taking longer to respond to the 2-shared conjunctive display toward the end of the single-target sessions - and correspondingly experienced more success at locating its target-this increase was not sufficient to compensate entirely for the greater difficulty of these particular displays.
Figure 6 summarizes the changes in RT across the single- and multiple-target conditions between the feature and conjunctive displays (only the results with the 2-shared condition are included for the three-dimensional set). The figure shows that during the multiple-target condition, when the accuracy difference between feature and conjunctive displays was the greatest, the difference between feature and conjunctive RTs was small. On the other hand, at the end of the single-target conditions, when the accuracy difference between these display types was markedly reduced or eliminated, the difference in RT had tangibly increased. One final analysis was conducted to see whether this apparent speed/accuracy tradeoff was confined to the test displays or was more general in nature, affecting the processing of the baseline displays as well. Evaluations of mean baseline performance over the four four-session groupings of the single-target sessions revealed no significant changes in accuracy $(69.2 \%, 69.2 \%, 70.7 \%$, and $70.8 \%$ ). First-peck RTs to baseline displays did show an 


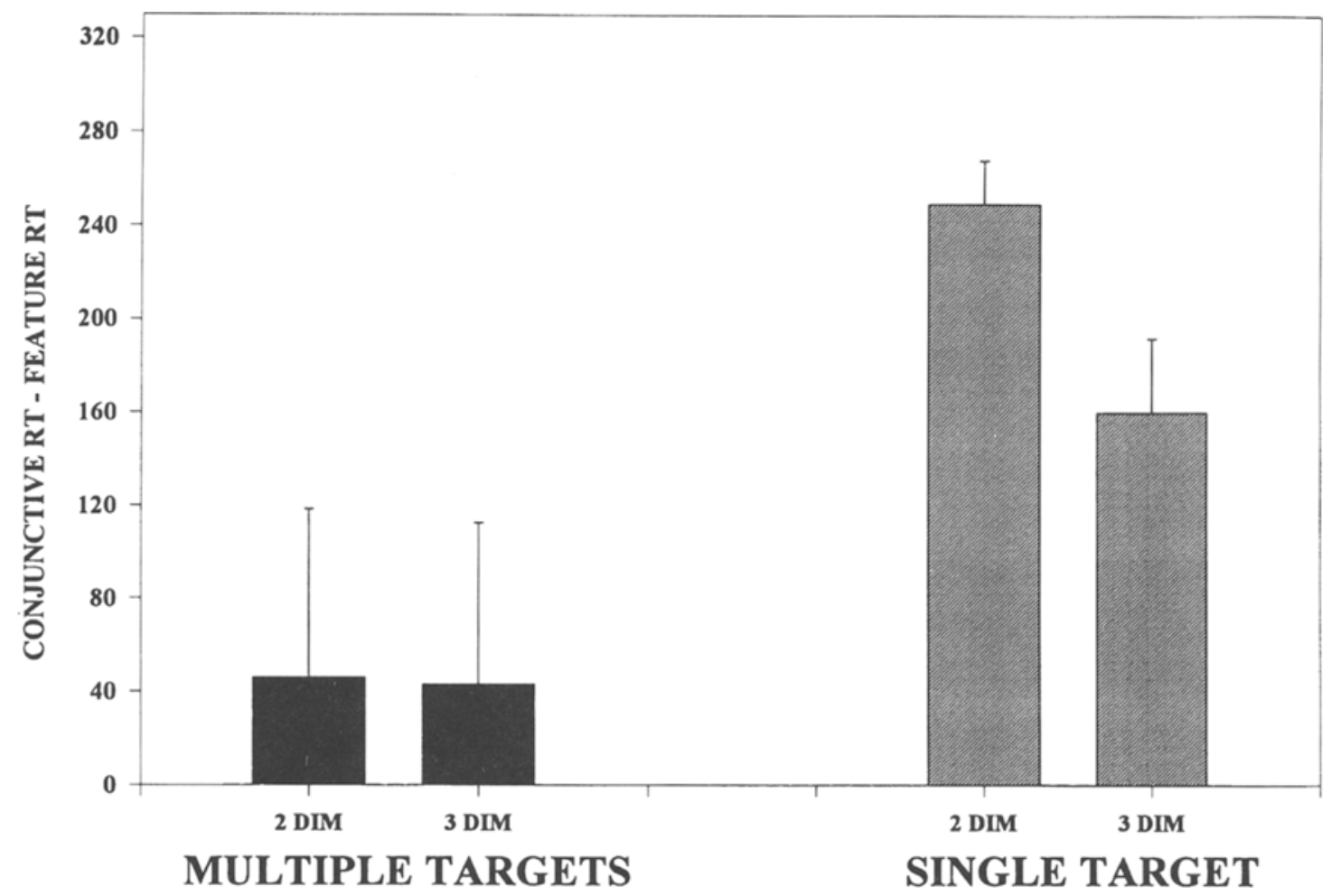

Figure 6. Mean difference in first-peck $R T$ between the feature and most difficult conjunctive display of the two( 2 DIM) or three- (3 DIM) dimensional sets during the third multiple-target phase and last four sessions of the three single-target phases of Experiment 2.

increase over the single-target sessions $(776,773,790$, and $821 \mathrm{msec}$ ), but no statistical foundation for this increase could be established $[F(3,15)=1.6]$. As such, the effects of target repetition on RT and accuracy appeared to be limited to the test displays.

\section{Discussion}

The important new result of Experiment 2 was the tradeoff discovered between the speed and accuracy of the pigeons' responses to the conjunctive displays. Whenever the pigeons delayed their initial responses to the conjunctive displays, their localization accuracy approached that for the feature displays. Correspondingly, whenever they responded rapidly to the conjunctive displays, their localization accuracy always fell considerably in comparison with that for the feature displays. This pattern suggests that pigeons can compensate for the perceptual difficulty of preattentively discriminating conjunctive target/distractor organizations, but as with humans, this costs additional processing time. The critical factor in changing this behavior seemed to be tied to the number of different targets used to form the test displays across sessions. When the test displays' target changed on a daily basis, differences in performance with the feature and with the conjunctive displays were exclusively in terms of accuracy. It was only when the same target was repeatedly tested over sessions that any difference in RT materialized. How did target repetition produce this change in search behavior?
One possibility is that repetition permits the target's identity to be known with greater certainty. Awareness of the target's identity strongly influences human visual search (Treisman \& Sato, 1990). One proposed mechanism for how this knowledge helps in conjunctive search has involved the top-down attentional modification of different sets of preattentive feature maps (Cave \& Wolfe, 1990; Treisman \& Sato, 1990). These proposals suggest the subject's knowledge about the target's dimensional attributes can be used to selectively examine only a subset of a conjunctive display's features, thereby producing faster search times by functionally reducing the task to a form of feature search (Egeth, Virzi, \& Garbart, 1984; Nakayama \& Silverman, 1986; Treisman \& Sato, 1990; Wolfe, 1992; Wolfe et al., 1989; see Reid \& Shettleworth, 1992, for a similar proposal for pigeons). Since our pigeons' RTs increased, rather than decreased, this type of feature-selection mechanism seems not to be the means by which target repetition influenced performance.

A popular mechanism for explaining similar RT effects in humans has relied on the metaphor of an "attentional spotlight" (Treisman \& Gormican, 1988). In this account, subjects are forced to focus this "spotlight" onto smaller portions of difficult displays in order to determine the target's presence and location, resulting in longer search times with such displays. This account easily accommodates the pigeons' increased RT and accuracy with conjunctive displays. However, this type of attentional-based 
strategy should be equally effective with all types of displays, and as such, it does not explain why the present tradeoff was essentially, if not exclusively, limited to the test displays, or why it depended on the number of targets being tested. This display specificity suggests that an alternative or additional mechanism needs to be considered.

Perhaps what underlies these effects involves a change in the pigeons' working description of what constitutes a "target," with repetition allowing a more detailed description of its properties to be generated and used than is normally employed with the baseline displays. Because of the large number and unpredictable nature of the targets appearing in the baseline displays, the birds' trial-to-trial representations of what constitutes a target must be a general one, one emphasizing the relational "differences" rather than the absolute values of the baseline displays (Cook, 1992a, 1993b). Consistent with this reasoning, for instance, is the excellent transfer to novel stimuli exhibited by pigeons in different forms of texture discrimination tasks (Cook, 1992a; Cook, Cavoto, \& Cavoto, 1995). If feature and conjunctive displays are tested when the birds are employing this generalized target description, the salient regions of the feature displays match this description better than the perceptually camouflaged conjunctive displays, resulting in the accuracy differences seen between these conditions most of the time (Experiment 1; multiple-target phase of Experiment 2, Cook, 1992b).

If frequently repeating a target results in establishing a more detailed internal description of its properties, the birds may be able to scan and successfully reject more portions of conjunctive displays, resulting in an increase in both accuracy and first-peck RT. Because the salient feature displays can still be detected preattentively, RTs to these displays would be affected little by repetition, although accuracy might be expected to increase because of the better match between a bird's current perception of the target and its internal memory for it. This greater specification of the test displays' target would be of no help of course on the still everchanging baseline trials. It might even be a temporary hindrance, at least until the birds resorted to using their more generalized target representation. Something like this may have happened on the baseline trials, as accuracy did not change, but RT appeared to increase slightly in the later stages of the target repetition sessions (although no statistical evidence for the latter change was established).

How did the birds know when to appropriately employ these specific and generalized target representations? One possibility is the general context of the display. The line elements and dimensional properties used to make the test displays did give them an instantly recognizable "look and feel" that was quite distinct from the baseline displays. Perhaps the birds learned to use their more detailed representations of the repeated targets only when the "context" was appropriate. If so, this account of display specificity shares much with the conditional expectancy hypothesis recently proposed by D. S. Blough (1993) to account for how the relative frequency of target/distractor combinations influences visual search in pigeons.
Finally, these results seem also to speak to recent discussions about how successive encounters with cryptic prey modify an animal's visual search behavior in more natural settings (P. M. Blough, 1991, 1992; Bond, 1983; Bond \& Riley, 1991; Gendron, 1986; Guilford \& Dawkins, 1987; Pietrewicz \& Kamil, 1977, 1979; Reid \& Shettleworth, 1992). Typically, repeated encounters with a particular cryptic prey increases a predator's subsequent capture rate for this prey for a period of time. In general, two classes of mechanisms have been proposed to explain this effect. "Search image" hypotheses propose that high encounter rates enhance the detectability of the cryptic prey by directly modifying the predator's discriminative processes (Bond, 1983; Tinbergen, 1960). These hypotheses predict that as prey abundance increases, accuracy should increase and search time or RT should decrease. "Search rate" hypotheses, in contrast, propose that predators adjust the amount of time that they spend looking for cryptic prey in order to optimize the accuracy of their search, given a prey's density. These hypotheses predict increased accuracy and increased search times when predators are looking for abundant cryptic prey (Gendron \& Staddon, 1983; Guilford \& Dawkins, 1987).

Given these contrasting predictions, the speed/accuracy tradeoffs observed in Experiments 1 and 2 are most compatible with the search rate hypotheses - where increases in capture rate with abundant cryptic prey (increases in localization accuracy with repeated conjunctive targets) occur only when search time increases (occur only when first-peck RT increases). However, Guilford and Dawkins (1987) argue that the search rate hypothesis predicts that once an animal has strategically adjusted its search threshold for a particular prey type, it should be just as successful with any equally cryptic prey. Given this perspective, our pigeons' success in slowing down for test displays in the single-target condition should have carried over to the equally discriminable test displays of the multiple-target conditions as well. The large drop in accuracy recorded at each of the single-target/multiple-target transitions indicates that this was not the case in the present experiment, which suggests that the birds were not simply adjusting a single generalized search threshold as in Guilford and Dawkins's account. This type of search specificity suggests instead important roles for both stimulus context and working memory in determining the speed and accuracy of a foraging animal's visual search behavior.

\section{EXPERIMENT 3}

In Experiment 3, we examined how the number of distractors in the feature and conjunctive test displays influenced performance. Recall that the human search for feature targets is rapid regardless of the number of distractors in the display, while the search duration for conjunctive targets systematically increases as the number of distractors increases (Treisman \& Gelade, 1980). Would the pigeons be similarly affected by the number of surrounding distractors in the present displays? Toward this end, we varied the number of distractors surrounding the target re- 
gions of both test and baseline displays in Experiment 3, reducing their number by $100 \%, 70 \%, 50 \%, 30 \%$, or $0 \%$ in different sessions. Because measurement of target localization accuracy seemed to capture the same information as did RT, but appeared to be the more sensitive and stable of the two measures with our birds, we employed four different targets in constructing the test displays of Experiment 3 in order to promote accuracy rather than RT differences among these displays.

\section{Method}

Subjects and Apparatus. The same 6 pigeons and apparatus were used. The experiment was conducted 2.5 months after Experiment 2 .

Procedure. The baseline and test displays were constructed in the same manner as in the previous experiments, with two exceptions: the baseline displays were now randomly constructed from an expanded pool of 21 total shapes (see Figure 1) and the .75-cm interelement spacing in the displays was varied by randomly adding 0,1 , or 2 pixels horizontally and/or vertically to each element's placement. The resulting misalignment helped break up the periodic structure of the displays. One of four target regions (BHS, GHL, BVL, or GVS elements) formed the test displays in any one session. These four targets were tested in a random order in four-session blocks that were varied independently of the manipulation of distractor number.

The number of distractors surrounding the target region of test and baseline displays was varied across sessions but was fixed for all trials within a session. The number of distractors tested across sessions represented $0 \%, 30 \%, 50 \%, 70 \%$, or $100 \%$ of the 342 distractors normally used $(0,102,171,240$, and 342 elements, respectively). For all displays in a session, the target region was first randomly located in the $24 \times 16$ matrix; then the remaining cells of the matrix were randomly filled in by the number of distractors scheduled for that session.

Each daily session consisted of 140 trials, testing 120 randomly selected baseline displays ( 60 color and 60 shape), the identical set of 18 test displays as described in Experiment 2, and two uniform displays. These uniform displays were created just as were the test and baseline displays, with a $7 \times 6$ "target" region of a randomly selected element randomly surrounded by different numbers of otherwise identical "distractor" elements. Thus, these uniform displays not only functioned as before to estimate "chance" target localization, but also provided information about how nondimensional factors, such as the target region's larger size and shape relative to that of the more sparsely distributed and discrete distractor elements, might have contributed to performance at the different distractor densities. Four five-session blocks testing all five distractor values in a random order were conducted for each bird. Following these sessions, eight additional sessions were conducted alternately testing the $50 \%$ and $70 \%$ distractor values. No differences were found between these two phases, and their results are combined below.

\section{Results}

Displayed in the three panels of Figure 7 are the accuracy data for the color and shape baseline displays (top panel), the two-dimensional set of feature and conjunctive displays (middle), and the three-dimensional set of feature and conjunctive displays (bottom). Overall, these panels show that increasing the number of distractors generally reduced accuracy in all types of displays. RT analyses found few differences among the conditions. There were no significant RT differences between the feature $(668 \mathrm{msec})$ and conjunctive ( $701 \mathrm{msec})$ displays overall, or as a function of distractor number $\left[F_{\mathrm{S}}(4,20)<2\right.$; this analysis used a com- bination of two- and three-dimensional data]. For the baseline displays, RT did significantly increase with distractor number $[0 \%=610,30 \%=613,50 \%=643,70 \%=695$, $100 \%=711 \mathrm{msec} ; F(4,20)=3.4]$. The longer RTs did not appear to benefit accuracy, however, as it declined across these same distractor values. Because the birds were responding quickly to each of the display types $(<715 \mathrm{msec})$, our remaining analyses focused on accuracy.

The best fitting line summarizing the results obtained with the "uniform" displays is repeated for reference in each of the panels of Figure 7 (the individual data points were excluded for graphic clarity). When no or very few individual "distractors" surrounded the larger, but dimensionally identical, "target" regions, accuracy was quite good with these displays. As more distractors were added, however, accuracy systematically declined to the chance levels anticipated on the basis of the two previous experiments (24.1\% during Experiment 3 ).

Although increasing the number of distractors reduced accuracy overall, the form of this interference strongly depended on a display's dimensional organization. Displays containing unidimensional target/distractor contrasts (color and shape baseline displays; size, color, and orientation feature displays) showed a different pattern of distractor effects than did the three types of conjunctive displays. For the baseline and feature displays, any interference caused by the distractors seemed to level off at the largest distractor values, while accuracy with the conjunctive displays continued to decline over the same values.

Linear and quadratic polynomial trend analyses were conducted on the results from each of these display types in order to examine how accuracy changed as a function of distractor number. Significant linear components were found for all display types, confirming the overall conclusion that increasing distractor number caused systematic declines in accuracy [all seven $F \mathrm{~s}(1,5)>11.4$ ]. Of far more interest was the presence of significant quadratic components in the birds' performance with all displays having a unidimensional contrast: the color baseline $[F(1,5)=$ 40.4], shape baseline $[F(1,5)=250.4]$, two-dimensional feature displays $[F(1,5)=6.7]$, and three-dimensional feature displays $[F(1,5)=9.5]$. No significant quadratic trends were found for the conjunctive conditions. These analyses confirm that accuracy linearly declined with the addition of more and more distractors to the conjunctive displays, but did not do so for the unidimensional feature and baseline displays. These contrasting trends are summarized by the linear or quadratic functions fitted to each display type's data points in Figure 7.

\section{Discussion}

The number of distractors present in the displays affected the birds' behavior. The most pronounced effect was the decrease in accuracy as distractor number increased. Such a generalized interference effect was expected, given the previous research on single-item visual search in pigeons (Allan \& D. S. Blough, 1989; D. S. Blough, 1977, 1979; P. M. Blough, 1984). Of greater theoretical interest 

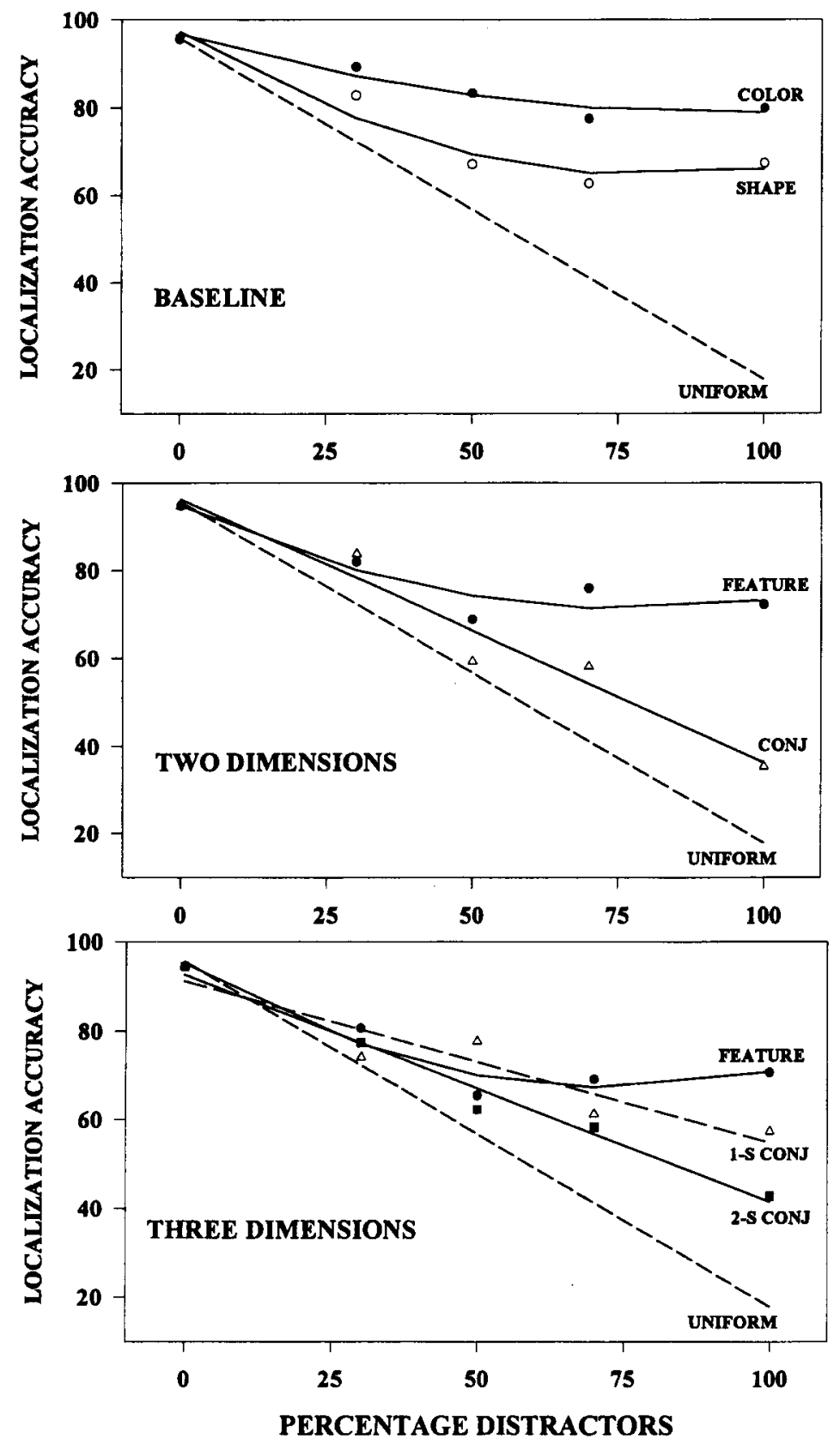

Figure 7. Mean target localization accuracies as a function of distractor number for baseline, feature, conjunctive, and uniform displays in Experiment 3 (CONJ = standard conjunctive; 1-S CONJ = 1-shared conjunctive; 2$S$ CONJ = 2-shared conjunctive). The curve for each condition represents the best-fitting polynomial equation as determined by the trend analyses described in the text.

was the new finding that this interference was qualitatively modified by the display's dimensional organization. The results with feature and baseline displays revealed that additional distractors initially caused a decline in accuracy, but that this effect disappeared as distractor density increased, with accuracy showing no further decline (and perhaps a slight increase) at the highest values tested. Accuracy with conjunctive displays, in contrast, simply declined in a linear fashion as further distractors were added. Collectively, the results suggest two sources of distractor interference on pigeon target localization.

When only a few scattered individual distractors were present, targets in all displays were easily located by the birds. This was surely because of the target's larger global appearance at these low distractor densities. That the target's size, or some correlated attribute, was sufficient for 
this discrimination is indicated by the high levels of "target"directed behavior in the uniform displays. This result suggests that the dimensional contrasts present in baseline and test displays probably made only minimal contributions to target localization at the very lowest distractor densities. This suggests that one source of distractor interference must have been on this nondimensional capacity to recognize the target region.

As more distractors were added, rendering this nondimensional cue increasingly useless, a second displaydependent source of interference emerged. Localization of the unidimensional targets of the baseline and feature displays became immune to further increases in distractor number, while conjunctive targets became only increasingly camouflaged. Thus, at these higher densities, the pattern of interference effects across the displays for the pigeons effectively parallels those seen in human feature and conjunctive search - with the discrimination of unidimensional contrasts being relatively independent of the number of distractors in the display, whereas performance with conjunctive organizations is highly dependent on the number of distractors present.

\section{GENERAL DISCUSSION}

The single best summary of these experiments is that the pigeons' discriminative responses to these different forms of multidimensional textured stimuli were strikingly humanlike. Experiment 1 established an analogous role for target/ distractor similarity in both species' performance with feature and conjunctive displays composed of two and three dimensions. In Experiment 2, with familiar targets, pigeons showed human-like RT differences in their processing of feature and conjunctive displays. Finally, Experiment 3 established a parallel effect of distractor number on both species' performance with feature and conjunctive displays. Whereas our earlier research had suggested a resemblance between pigeons and humans limited to the superior discrimination of feature displays over conjunctive displays, the results from these three more exacting experiments make the case for this resemblance even more compelling by expanding the number and nature of these behavioral similarities.

The first implication of these comparable outcomes is that they suggest that pigeons and humans see these complex multidimensional stimuli similarly. The second, and perhaps more contentious, implication is that they suggest that identical or functionally equivalent psychological mechanisms are used by both species to process textured dimensional structure. Of course, despite the manifest similarities, the possibility remains that our textured feature and conjunctive analogues are not tapping the same underlying processes in pigeons as in human feature and conjunctive visual search. Although we think this an unlikely alternative, a replication of the present studies with birds trained and tested in a multidimensional single-target visual search task would be a welcome confirmation.

Overall, we believe that a multistage model of visual processing similar to those proposed for humans explains these results best. We propose that multielement displays are initially encoded by the pigeons with parallel sets of feature-based operators that are arranged in separate and independent dimensional channels. So far, the evidence suggests that color, size, and orientation all function in this capacity (Experiment 1; Cook, 1992b). Within these dimensional channels, highly similar, closely spaced feature information is then perceptually grouped into visual regions. This grouping operation establishes the location and magnitude of the regularities and contrasts present in each dimension of the stimulus. Furthermore, this perceptual grouping and segregation is simultaneously executed at several different spatial scales, ranging from small local differences between individual elements to larger scale global differences between target/distractor regions. The information derived from the smaller scales is employed in detecting the fine details of the visual world, while the larger scale groupings allow emergent information about global discontinuities and regularities within and between objects to be quickly derived (Cook, 1993b). Fixed and odditem visual search tasks involving only a single target element probably emphasize the use of smaller scale local differences by the pigeons, while the current texture discriminations encourage the discrimination of the global or regional differences in the displays. The birds' allocation of spatial "attention" to these different scales is probably only one of emphasis, however, because each scale probably makes ancillary contributions to each of these types of visual discriminations - sometimes allowing global oddity to influence the search for single targets (D. S. Blough, 1989) or the identity of local elements to influence texture discrimination (Cook, 1992a).

The next stage in processing combines the information from the dimensional channels into a common map of the differences present in all of the dimensions, again at several different spatial scales. This integration is automatic (i.e., nonattentional) and topographic in organization. This integrative step is suggested by the direct relation between the accuracy of target localization and the degree of feature overlap present in the different regions of the display. For instance, performance with the redundant displays improved as the number of dimensional contrasts increased. This redundancy gain is consistent with the idea that the separate dimensional signals are being combined in some fashion prior to responding. The systematic change in conjunctive performance as a function of feature overlap carries much the same implication, although in this case the integration of the weak and inconsistent "difference" signals from each dimension results in quite poor target detection.

Until the present experiments, this type of bottom-up, difference-oriented discrimination mechanism easily accounted for the majority of our results with textured stimuli. Given the highly variable and large number of regional contrasts regularly encountered by the pigeons in these procedures, it is perhaps not too surprising that their targetdirected behavior came to be primarily controlled by the invariant regional differences present in these displays (Cook, 1992a, 1992b, 1992c, 1993b). This model cannot 
account, however, for the speed/accuracy tradeoffs detected with the conjunctive displays in Experiments 1 and 2. In Experiment 2, a memory-based mechanism was proposed to account for the increased accuracy and RTs observed in that experiment with familiar targets. For such a mechanism to work, however, a greater specification of the display's dimensional properties must be available than can be provided by a simple "difference" mechanism. The exact relation between the seemingly immediate recovery of unidimensional differences from these textured displays and the more detailed identification of their attributes as used by these top-down factors represent an important area for future research.

Readers familiar with the human texture segregation and visual search literature will immediately recognize many of these mechanisms and their thematic similarity to models of human multielement perception and search (e.g., Cave \& Wolfe, 1990; Hoffman, 1979; Poggio, Gamble, \& Little, 1988; Watt, 1988). The reason is simple. Despite the large differences in the size and neural organization of the human and pigeon visual systems (Pearson, 1972), the present experiments suggest that the visual mechanisms for processing dimensional information are very similar in these two species. If so, it may be a good example of the independent evolution of computationally convergent processes in different neural architectures, suggesting that universal rules or constraints may exist regarding the processing of object-related visual information across species (Shepard, 1984). Alternatively, it could mean that these dimensional processing mechanisms are very old, evolving prior to the reptilian split 200 million years ago leading to today's birds and mammals.

\section{REFERENCES}

ALlan, S. E., \& Blough, D. S. (1989). Feature-based search asymmetries in pigeons and humans. Perception \& Psychophysics, 46, 456-464.

AsHBY, F. G. (ED.) (1992). Multidimensional models of perception and cognition. Hillsdale, NJ: Erlbaum.

BECK, J. (1966). Effect of orientation and of shape similarity on perceptual grouping. Perception \& Psychophysics, 1, 300-302.

BECK, J. (1982). Textural segmentation. In J. Beck (Ed.), Organization and representation in perception (pp. 285-318). Hillsdale, NJ: Erlbaum.

BLough, D. S. (1977, May 27). Visual search in pigeons: Hunt and peck method. Science, 196, 1013-1014.

BLOUGH, D. S. (1979). Effects of number and form of stimuli on visual search in the pigeon. Journal of Experimental Psychology: Animal Behavior Processes, 5, 211-223.

Blough, D. S. (1989). Odd-item search in pigeons: Display size and transfer effects. Journal of Experimental Psychology: Animal Behavior Processes, 15, 14-22.

BLough, D. S. (1993). Effects on search speed of probability of targetdistractor combinations. Journal of Experimental Psychology: Animal Behavior Processes, 19, 231-243.

Blovgh, P. M. (1984). Visual search in pigeons: Effects of memory set size and display variables. Perception \& Psychophysics, 35, 344352.

BLough, P. M. (1991). Selective attention and search images in pigeons. Journal of Experimental Psychology: Animal Behavior Processes, 17, 292-298.

BLougH, P. M. (1992). Detectability and choice during visual search: Joint effects of sequential priming and discriminability. Animal Learning \& Behavior, 20, 293-300.

BoND, A. B. (1983). Visual search and selection of natural stimuli in the pigeon: The attention threshold hypothesis. Journal of Experimental Psychology: Animal Behavior Processes, 9, 292-306.

BoND, A. B., \& RILEY, D. A. (1991). Searching image in the pigeon: A test of three hypothetical mechanisms. Ethology, 87, 203-224.

BRown, M. F. (1987). Dissociation of stimulus compounds by pigeons. Journal of Experimental Psychology: Animal Behavior Processes, 13, 80-91.

CAVE, K. R., \& WolfE, J. M. (1990). Modeling the role of parallel processing in visual search. Cognitive Psychology, 22, 225-271.

CooK, R. G. (1992a). Acquisition and transfer of visual texture discriminations by pigeons. Journal of Experimental Psychology: Animal Behavior Processes, 18, 341-353.

Cook, R. G. (1992b). Dimensional organization and texture discrimination in pigeons. Journal of Experimental Psychology: Animal Behavior Processes, 18, 354-363.

CoOK, R. G. (1992c). The visual perception and processing of textures by pigeons. In W. K. Honig \& G. Fetterman (Eds.), Cognitive aspects of stimulus control (pp. 279-299). Hillsdale, NJ: Erlbaum.

CoOK, R. G. (1993a). The experimental analysis of cognition in animals. Psychological Science, 4, 174-178.

Cook, R. G. (1993b). Gestalt contributions to visual texture discriminations by pigeons. In T. Zentall (Ed.), Animal cognition: A tribute to Donald A. Riley (pp. 251-270). Hillsdale, NJ: Erlbaum.

Cook, R. G., Cavoto, K. K., \& Cavoto, B. R. (1995). Same/different texture discrimination and concept learning in pigeons. Journal of Experimental Psychology: Animal Behavior Processes, 21, 253-260.

DEHAENE, S. (1989). Discriminability and dimensionality effects in visual search for featural conjunctions: A functional pop-out. Perception \& Psychophysics, 46, 72-80.

DUNCAN, J., \& HuMPHREYS, G. W. (1989). Visual search and stimulus similarity. Psychological Review, 96, 433-458.

Egeth, H., VirzI, R. A., \& Garbart, H. (1984). Searching for conjunctively defined targets. Journal of Experimental Psychology: Human Perception \& Performance, 10, 32-39.

GARNER, W. R. (1974). The processing of information and structure. Potomac, MD: Erlbaum.

GARNER, W. R. (1976). Interaction of stimulus dimensions in concept and choice processes. Cognitive Psychology, 8, 98-123.

GENDRON, R. P. (1986). Searching for cryptic prey: Evidence for optimal search rates and the formation of search images in quail. Animal Behaviour, 34, 898-912.

Gendron, R. P., \& STaddon, J. E. R. (1983). Searching for cryptic prey: The effect of search rate. American Naturalist, 121, 172-186.

GuILFORD, T., \& Dawkins, M. S. (1987). Search images not proven: A reappraisal of recent evidence. Animal Behaviour, 35, 1838-1845.

HoffMAn, J. E. (1979). A two-stage model of visual search. Perception \& Psychophysics, 25, 319-327.

HoNiG, W. K. (1991). Discrimination by pigeons of mixture and uniformity in arrays of stimulus elements. Journal of Experimental Psychology: Animal Behavior Processes, 17, 68-80.

JuLESz, B. (1981). Textons, the elements of texture perception and their interactions. Nature, 290, 91-97.

MADDOX, T. W. (1992). Perceptual and decisional separability. In F. G. Ashby (Ed.), Multidimensional models of perception and cognition (pp. 147-180). Hillsdale, NJ: Erlbaum.

MARR, D. (1982). Vision. San Francisco: W. H. Freeman.

Melara, R. D., \& Marks, L. E. (1990). Perceptual primacy of dimensions: Support for a model of dimensional interaction. Journal of Experimental Psychology: Human Perception \& Performance, 16, 398-414.

NaKayama, K., \& Silverman, G. (1986). Serial and parallel processing of visual feature conjunctions. Nature, 320, 264-265.

PACHELla, R. G. (1974). The interpretation of reaction time in information processing research. In B. Kantowitz (Ed.), Human information processing: Tutorials in performance and cognition (pp. 41-82). New York: Halstead.

PASHler, H. (1988). Cross-dimensional interaction and texture segregation. Perception \& Psychophysics, 43, 307-318.

PeARSON, R. (1972). The avian brain. New York: Academic Press.

Pietrewicz, A. T., \& Kamil, A. C. (1977, February 11). Visual detection of cryptic prey by blue jays (Cyanocitta cristata). Science, 195 , 580-582. 
Pietrewicz, A. T., \& Kamil, A. C. (1979, June 22). Search image formation in the blue jay (Cyanocitta cristata). Science, 204, 1332-1333.

Poggio, T., Gamble, B., \& Little, J. J. (1988, October 21). Parallel integration of vision modules. Science, 242, 436-439.

Quinlan, P. T., \& Humphreys, G. W. (1987). Visual search for targets defined by combinations of color, shape, and size: An examination of the task constraints on feature and conjunction searches. Perception \& Psychophysics, 41, 455-472.

Reid, P. J., \& ShetTleworth, S. J. (1992). Detection of cryptic prey: Search image or search rate? Journal of Experimental Psychology: Animal Behavior Processes, 17, 273-286.

Riley, D. A., \& LeITH, C. R. (1976). Multidimensional psychophysics and selective attention in animals. Psychological Bulletin, 83, 138-160.

SHEPARD, R. N. (1984). Ecological constraints on internal representation: Resonant kinematics of perceiving, imagining, thinking and dreaming. Psychological Review, 91, 417-447.

Tinbergen, L. (1960). The natural control of insects in pinewoods: I. Factors influencing the intensity of predation by song birds. Archives Neerlandaises de Zoologie, 13, 265-343.

Treisman, A. (1988). Features and objects: The Fourteenth Barlett
Memorial Lecture. Quarterly Journal of Experimental Psychology, 40A, 201-237.

Treisman, A., \& Gelade, G. (1980). A feature-integration theory of attention. Cognitive Psychology, 12, 97-136.

Treisman, A., \& Gormican, S. (1988). Feature analysis in early vision: Evidence from search asymmetries. Psychological Review, 95, 15-48.

Treisman, A., \& Sato, S. (1990). Conjunction search revisited. Journal of Experimental Psychology: Human Perception \& Performance, 16, 459-478.

WATT, R. (1988). Visual processing. Hove, U.K.: Erlbaum.

WoLfE, J. M. (1992). "Effortless" texture segmentation and "parallel" visual search are not the same thing. Vision Research, 32, 757-763.

Wolfe, J. M., CAVE, K. R., \& Franzel, S. L. (1989). Guided search: An alternative to the feature-integration model for visual search. Journal of Experimental Psychology: Human Perception \& Performance, 15, 419-433.

(Manuscript received July 20, 1994; revision accepted for publication February 27,1995 .) 